\title{
A study on the determinants of some Hessenberg Toeplitz Bohemians
}

\author{
Jishe Feng $^{1, \mathrm{a}}$, Hongtao Fan ${ }^{\mathrm{b}}$ \\ ${ }^{a}$ School of Mathematics and Statistics, Longdong University, Qingyang, Gansu, \\ 745000, PR China \\ ${ }^{\mathrm{b}}$ College of Science, Northwest A\&University, Yangling, Shaanxi 712100, PR \\ China
}

\begin{abstract}
In this paper, we deduce explicit formulas to evaluate the determinants of nonsymmetrical structure Toeplitz Bohemians by two determinants of specific Hessenberg Toeplitz matrices, which are linear combinations in terms of determinants of specific Hessenberg Toeplitz matrices. We get some new results very different from Massimiliano Fasi and Gian Maria Negri Porzio's papers.
\end{abstract}

2000 Mathematics Subject Classification: 11C20; 15B36; 15A18; 15B05

Key words: Bohemian; Toeplitz matrix; Hessenberg matrix; tridiagonal matrix; pentadiagonal matrix

\section{Introduction}

Bohemian which comes from an abbreviation of BOunded HEight Matrix of Integers, is the matrix whose elements are drawn from a fixed finite integers [3]. Bohemians are both useful and interesting in several research areas. Researchers have studied Bohemians from the $50 \mathrm{~s}$ in the last century [4]. An overview of recent results and conjectures about Bohemians gives on the website [25]. Almost all of the researchers specialize Bohemians to Hessenberg Toeplitz matrices. Tridiagonal and Pentadiagonal are specific Hessenberg matrices that frequently appear in matrix algebra, spectral analysis, statistics, differential equations, etc., and are of significance in several research areas.

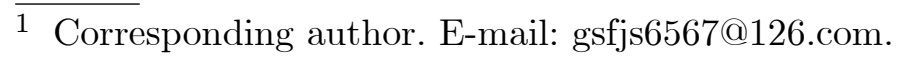


For further details, see [7-12, 26] and the references therein. Thornton [25] has proposed many conjectures to request the numbers of distinct determinants of $n \times n$ Hessenberg Bohemians.

In matrix calculation, ones are used to matrix multiplication or decomposition, for instance, the $Q R, L U$ factorization. If a matrix has a specific structure, such as symmetric, tridiagonal, pentadiagonal, heptadiagonal, etc. one utilizes these properties to compute those determinants. It is well known [13] a general real matrix $X$ can be decomposed to

$$
X=U Y U^{T}
$$

where $U$ is orthogonal, and $Y$ is an upper Hessenberg matrix. There is a result for the determinants $|X|=|Y|$. It is difficult to find the matrix $Y$, especially, when matrix $X$ is a Bohemian, matrix $U$ and $Y$ are not Bohemians in most cases, the error is too huge to compute the determinants $U$ and $Y$.

Recently Andelic and Fonseca [22] considered how to evaluate the determinants of the pentadiagonal Toeplitz matrix and proposed a research problem: "Find the determinant of a pentadiagonal Toeplitz matrix, where the four subdiagonals are not necessarily immediately below (two) and above (two) the main diagonal or are not consecutive." They proposed two conjectures in the cases of pentadiagonal matrices containing one or two entries in the second subdiagonal above the main diagonal. By the Laplace expansion formula and the elementary transformation, the authors proved that the conjectures are true and gave two new results [11].

In this paper, we propose the determinants of any nonsymmetric Toeplitz matrices, which are Bohemians with elements from $\{-1,0,1\}$ or $\{0,1\}$. By the Laplace expansion formula, we can deduce those determinants to the expressions in terms of the linear combinations of specific Toeplitz matrices. Namely, for $n, i$ and $k$ are positive integers. For an $n \times n$ Bohemian $X_{n}$, its determinant is $\left|X_{n}\right|$, and a specific matrix $Y_{n-i}$, its determinant is $\left|Y_{n-i}\right|, a_{i}$ $(i=0,1,2, \cdots)$ are combinatorial coefficients, There is

$$
\left|X_{n}\right|=a_{1}\left|Y_{n-1}\right|+a_{2}\left|Y_{n-2}\right|+\cdots+a_{k}\left|Y_{n-k}\right|
$$

In [9], $\left|Y_{n}{ }_{i}\right|=d(d+1)^{i-1}, a_{i}=(-1)^{n-i} \widetilde{a}_{i}$, and $\widetilde{a}_{i} \in\{-d,-d+1, \cdots, d-1, d\}$. Here we have three methods to derive the formula (2), one is to apply the Laplace expansion, one is to solve the recurrence relations, and the other one is to compute specific Hessenberg determinants. We also give some Hessenberg Toeplitz Bohemians to check the results in [9] and get some very different conclusions.

The rest of this paper is organized as follows: In Section 2, we give notations and recall four lemmas. In Section 3, we propose three explicit formulas to cal- 
culate the determinants of tridiagonal Toeplitz matrices. In Section 4, we deduce three explicit formulas to calculate the determinants of non-symmetrical structure pentadiagonal Toeplitz matrices. We generalize two explicit formulas to calculate the determinants of another non-symmetrical structure sixdiagonal Toeplitz matrices. In Section 5, we talk about the results in [9], give three remarks on the propositions and some conclusions.

\section{Notations and Lemmas}

Except as the specific statement, we follow the notations as in [22], all mentioned elements are 1s, all the unmentioned elements are zeros.

Lemma 1 [2] A lower Hessenberg matrix, $M_{n}=\left(m_{i, j}\right)$, is an $n \times n$ matrix such that $m_{i, j}=0$ whenever $j>i+1$ and $m_{i, i+1} \neq 0$ for some $i$, i.e.

$$
M_{n}=\left[\begin{array}{ccccc}
m_{11} & m_{12} & 0 & \cdots & 0 \\
m_{21} & m_{22} & m_{23} & \ddots & \vdots \\
m_{31} & m_{32} & m_{33} & \ddots & 0 \\
\vdots & \vdots & \ddots & \ddots & m_{n-1, n} \\
m_{n, 1} & m_{n, 2} & \cdots & m_{n, n-1} & m_{n, n}
\end{array}\right] .
$$

Let $\left|M_{0}\right|=1$ and $\left|M_{1}\right|=m_{11}$. Then there is the following formula of the determinant: for $n \geq 2$,

$$
\left|M_{n}\right|=m_{n, n} \cdot\left|M_{n-1}\right|+\sum_{k=1}^{n-1}\left((-1)^{n-k} m_{n, k} \prod_{j=k}^{n-1} m_{j, j+1} \cdot\left|M_{k-1}\right|\right) .
$$

Lemma 2 [8] Let $c_{1,} c_{2}, \cdots, c_{k}$ be real numbers. Suppose that the characteristic equation $r^{k}-c_{2} r^{k-1}-\cdots-c_{k}=0$ has $k$ distinct roots $r_{1}, r_{2}, \cdots, r_{k}$. Then, a sequence $\left\{a_{n}\right\}$ is a solution of the recurrence relation $a_{n}=c_{1} a_{n-1}+c_{2} a_{n-2}+$ $\ldots+c_{k} a_{n-k}$. If and only if

$$
a_{n}=\alpha_{1} r_{1}^{n}+\alpha_{2} r_{2}^{n}+\cdots+\alpha_{k} r_{k}^{n}
$$

For $n=0,1,2, \cdots$, where $\alpha_{1}, \alpha_{2}, \cdots, \alpha_{k}$ are constants.

Lemma 3 [8] Let $c_{1,} c_{2}, \cdots, c_{k}$ be real numbers. Suppose that the characteristic equation $r^{k}-c_{2} r^{k-1}-\cdots-c_{k}=0$ has $t$ distinct roots $r_{1}, r_{2}, \cdots, r_{t}$, with multiplicities $m_{1}, m_{2}, \cdots, m_{t}$ respectively, so that $m_{i} \geq 1$, for $i=1,2, \cdots, t$ and $m_{1}+m_{2}+\cdots+m_{t}=k$. Then, a sequence $\left\{a_{n}\right\}$ is a solution of the recurrence relation $a_{n}=c_{1} a_{n-1}+c_{2} a_{n-2}+\ldots+c_{k} a_{n-k}$. If and only if 


$$
\begin{aligned}
a_{n}= & \left(\alpha_{1,0}+\alpha_{1,1} n+\alpha_{1,2} n^{2}+\cdots+\alpha_{1, m_{1}-1} n^{m_{1}-1}\right) r_{1}^{n} \\
& +\left(\alpha_{2,0}+\alpha_{2,1} n+\alpha_{2,2} n^{2}+\cdots+\alpha_{2, m_{2}-1} n^{m_{2}-1}\right) r_{2}^{n} \\
& +\cdots+\left(\alpha_{t, 0}+\alpha_{t, 1} n+\alpha_{t, 2} n^{2}+\cdots+\alpha_{t, m_{t}-1} n^{m_{t}-1}\right) r_{t}^{n}
\end{aligned}
$$

For $n=0,1,2, \cdots$, where $\alpha_{i, j}$ are constants for $1 \leq i \leq t$ and $0 \leq j \leq m_{i}-1$.

Lemma 4 [11] Let the determinant $\left|H_{n}\right|$ of an $n \times n$ lower Hessenberg matrix

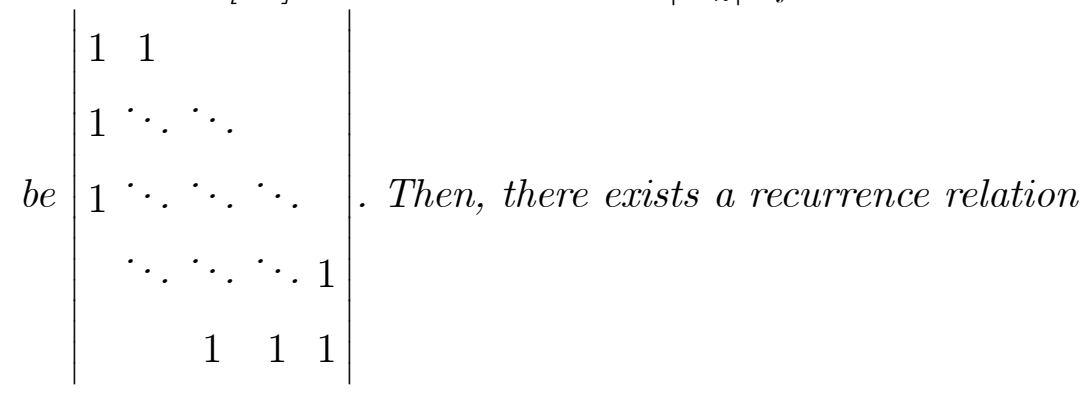

$$
\left|H_{n}\right|=\left|H_{n-1}\right|-\left|H_{n-2}\right|+\left|H_{n-3}\right|,
$$

and an explicit formula

$$
\left|H_{n}\right|=\frac{1}{2}+\frac{1-i}{4} i^{n}+\frac{1+i}{4}(-i)^{n}=\left\{\begin{array}{c}
1, \text { if } n \equiv 0,1(\bmod 4) \\
0, \text { if } n \equiv 2,3(\bmod 4)
\end{array}, \text { for } n \geq 0\right.
$$

where $i^{2}=-1$.

\section{The determinants of tridiagonal and their generalized Toeplitz matrices}

\subsection{The symmetrical matrices}

The $n \times n$ symmetrical structure tridiagonal matrix $T_{n}^{(k)}=\left(t_{i, j}\right)_{n \times n}$ is called $k$-tridiagonal Toeplitz in [12], its elements are

$$
t_{i, i}=\left\{\begin{array}{l}
1, i=j, j \pm k \\
0, \text { otherwise }
\end{array}\right.
$$


where $i, j=1,2, \cdots, n$, and $k$ is a prescribed positive integer. Especially, the 1-tridiagonal Toeplitz matrix is so-called tridiagonal matrix

$$
T_{n}^{(1)}=\left[\begin{array}{cccc}
1 & 1 & & \\
1 & \ddots & \ddots & \\
& \ddots & \ddots & 1 \\
& & 1 & 1
\end{array}\right]
$$

Theorem 5 For the determinant $\left|T_{n}^{(1)}\right|$ of an $n \times n$ tridiagonal matrix $T_{n}^{(1)}$, there exists a recurrence relation

$$
\left|T_{n}^{(1)}\right|=\left|T_{n-1}^{(1)}\right|-\left|T_{n-2}^{(1)}\right|
$$

and an explicit formula, for $n \geq 0$

$\left|T_{n}^{(1)}\right|=\frac{3+i \sqrt{3}}{6}\left(\frac{1-i \sqrt{3}}{2}\right)^{n}+\frac{3-i \sqrt{3}}{6}\left(\frac{1+i \sqrt{3}}{2}\right)^{n}=\left\{\begin{array}{rl}1, & \text { if } n \equiv 0,1(\bmod 6) \\ 0, & \text { if } n \equiv 2,5(\bmod 6) \\ -1, \text { if } n & \equiv 3,4(\bmod 6)\end{array}\right.$.

where $i^{2}=-1$.

PROOF. Because that determinant $\left|T_{n}^{(1)}\right|$ is a specific Hessenberg determinant, so we apply Lemma 1, and obtain the recurrence relation (9).

Applying Lemma 2 to (9), we obtain its characteristic equation $x^{2}=x-1$. Solving this equation, we obtain its roots $\frac{1-i \sqrt{3}}{2}$ and $\frac{1+i \sqrt{3}}{2}$, where $i^{2}=-1$.

By evaluating the determinants $\left|T_{4}^{(1)}\right|$ and $\left|T_{5}^{(1)}\right|$ via elementary operations, we obtain $\left|T_{4}^{(1)}\right|=-1$ and $\left|T_{5}^{(1)}\right|=0$.

According to Lemma 2, assume that $\left|T_{n}^{(1)}\right|=\alpha \lambda_{1}^{n}+\beta \lambda_{2}^{n}$, for $n=4,5$, we obtain the system of equations,

$$
\left\{\begin{array}{l}
\alpha\left(\frac{1-i \sqrt{3}}{2}\right)^{4}+\beta\left(\frac{1+i \sqrt{3}}{2}\right)^{4}=-1 \\
\alpha\left(\frac{1-i \sqrt{3}}{2}\right)^{5}+\beta\left(\frac{1+i \sqrt{3}}{2}\right)^{5}=0
\end{array}\right.
$$

By solving it, we obtain $\alpha=\frac{3+i \sqrt{3}}{6}, \beta=\frac{3-i \sqrt{3}}{6}$, thereby completing the proof of formula (10). 
As in [21], there is $\left|T_{n}^{(1)}\right|=U_{n}\left(\frac{1}{2}\right)$, where $U_{n}\left(\frac{1}{2}\right)$ is the value of the nth-degree Chebyshev polynomial of the second kind. Thus, we obtain a new formula about the Chebyshev polynomial of the second kind.

$$
U_{n}\left(\frac{1}{2}\right)=\frac{3+i \sqrt{3}}{6}\left(\frac{1-i \sqrt{3}}{2}\right)^{n}+\frac{3-i \sqrt{3}}{6}\left(\frac{1+i \sqrt{3}}{2}\right)^{n} .
$$

For the determinant of 2-tridiagonal Toeplitz matrix,

$$
T_{n}^{(2)}=\left[\begin{array}{ccccc}
1 & 0 & 1 & & \\
0 & \ddots & \ddots & \ddots & \\
1 & \ddots & \ddots & \ddots & 1 \\
& \ddots & \ddots & \ddots & 0 \\
& & 1 & 0 & 1
\end{array}\right]
$$

which has been studied in [22], [15], [20], [12]. Expanding the determinant by the last row and last column, we obtain a recurrence relation $\left|T_{n}^{(2)}\right|=$ $\left|T_{n}^{(2)}{ }_{1}\right|-\left|T_{n}^{(2)}{ }_{3}\right|+\left|T_{n}^{(2)}{ }_{4}\right|$, and its characteristic equation $x^{4}-x^{3}+x-1=0$.

Applying Lemma 2.2, we obtain

$$
\left|T_{n}^{(2)}\right|=\frac{1}{2}+\frac{(-1)^{n}}{6}+\frac{1+i \sqrt{3}}{6}\left(\frac{1-i \sqrt{3}}{2}\right)^{n}+\frac{1-i \sqrt{3}}{6}\left(\frac{1+i \sqrt{3}}{2}\right)^{n}=\left\{\begin{array}{l}
1, \text { if } n \equiv 0,1,2(\bmod 6) \\
0, \text { if } n \equiv 3,4,5(\bmod 6)
\end{array}\right.
$$

For the determinant of the 3-tridiagonal Toeplitz matrix,

$$
\left|T_{n}^{(3)}\right|=\left|\begin{array}{ccccc}
1 & 0 & 0 & 1 \\
0 & \ddots & \ddots & \ddots & 1 \\
0 & \ddots & \ddots & \ddots & 0 \\
1 & \ddots & \ddots & \ddots & 0 \\
1 & 0 & 0 & 1
\end{array}\right|
$$

After computing with the Maple, we obtain

$$
\left|T_{n}^{(3)}\right|=\left\{\begin{array}{l}
1, \quad \text { if } n \equiv 0,1,2,3(\bmod 18) \\
0, \quad \text { if } n \equiv 4,5,6,7,8,13,14,15,16,17(\bmod 18) \\
-1, \text { if } n \equiv 9,10,11,12(\bmod 18)
\end{array}\right.
$$

In general cases, we have the following theorem. 
Theorem 6 For the symmetrical structure $k$-tridiagonal Toeplitz matrices, namely the Bohemian which elements from $\{0,1\}$, its maximum absolute determinant is equal to 1. Especially, when the positive integer $k$ is odd, the set of determinant $\left|T_{n}^{(k)}\right|$ is $\{-1,0,1\}$. When $k$ is even, the set of determinant $\left|T_{n}^{(k)}\right|$ is $\{0,1\}$.

PROOF. Assume $n=k q-r$, then $n=k(q-1)+k-r$. Applying the relation $(D)$ in [1], we obtain

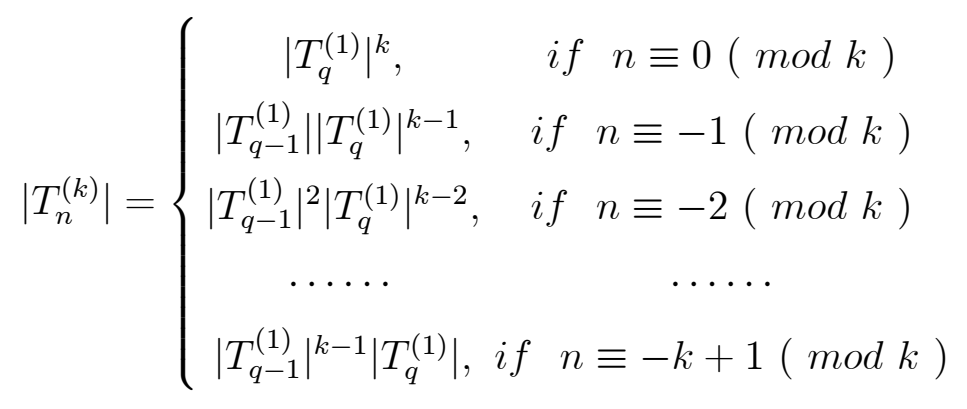

\begin{tabular}{|c|c|c|c|}
\hline$k$ & $r$ & $k-r$ & The powers of $\left|T_{q-1}^{(1)}\right|^{r}\left|T_{q}^{(1)}\right|^{k-r}$ \\
\hline odd & odd & even & odd \\
\hline odd & even & odd & odd \\
\hline even & odd & odd & even \\
\hline even & even & even & even \\
\hline
\end{tabular}

By the formula (4) and the last column in the above table, we complete the proof.

\subsection{Nonsymmetric structure}

For the determinant

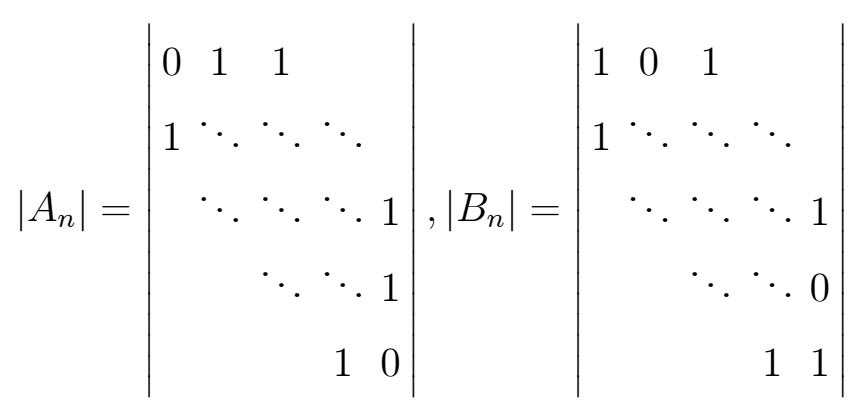


Applying Lemma 1 to the Hessenberg determinants, we have three-degree recurrence relations: $\left|A_{n}\right|=-\left|A_{n-2}\right|+\left|A_{n-3}\right|$ and $\left|B_{n}\right|=\left|B_{n-1}\right|+\left|B_{n-3}\right|$. Applying Lemma 2, we obtain their character equations as $x^{3}+x-1=0$ and $x^{3}-x^{2}-1=0$. Solving this two equations, we have three distinct roots, $x_{1}, x_{2}, x_{3}$, respectively. We omit them.

Using the above recurrence relations, we can compute the first some terms of two sequences as the followings

\begin{tabular}{|l|l|l|l|l|l|l|l|l|l|l|l|l|l|l|l|}
\hline $\mathrm{n}$ & 5 & 6 & 7 & 8 & 9 & 10 & 11 & 12 & 13 & 14 & 15 & 16 & 17 & 18 & 19 \\
\hline$\left|A_{n}\right|$ & 2 & 0 & -3 & -2 & -3 & 5 & 1 & -8 & 4 & 9 & -12 & -5 & 21 & -7 & -26 \\
\hline$\left|B_{n}\right|$ & 4 & 6 & 9 & 13 & 19 & 28 & 41 & 60 & 88 & 129 & 189 & 277 & 406 & 595 & 872 \\
\hline
\end{tabular}

\section{The determinants of Pentadiagonal and generalized Toeplitz ma- trices}

\subsection{The symmetrical pentadiagonal Toeplitz matrices}

For the determinant of the pentadiagonal Toeplitz matrix

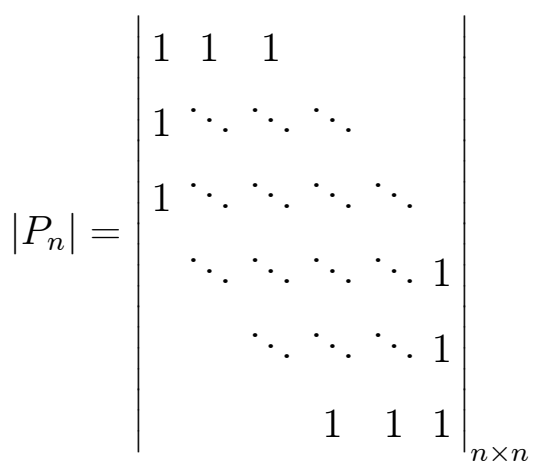

As the result of 7-term recurrence relation [24], [22], we know a recurrence relation $\left|P_{n}\right|=\left|P_{n-1}\right|+\left|P_{n-5}\right|-\left|P_{n-6}\right|$. By the software Maple, we obtain

$$
\left|P_{n}\right|=\left\{\begin{array}{l}
0, \text { if } n \equiv 2,3,4(\bmod 5) \\
1, \text { if } n \equiv 0,1 \quad(\bmod 5)
\end{array}\right.
$$


For the determinant of the pentadiagonal Toeplitz matrix

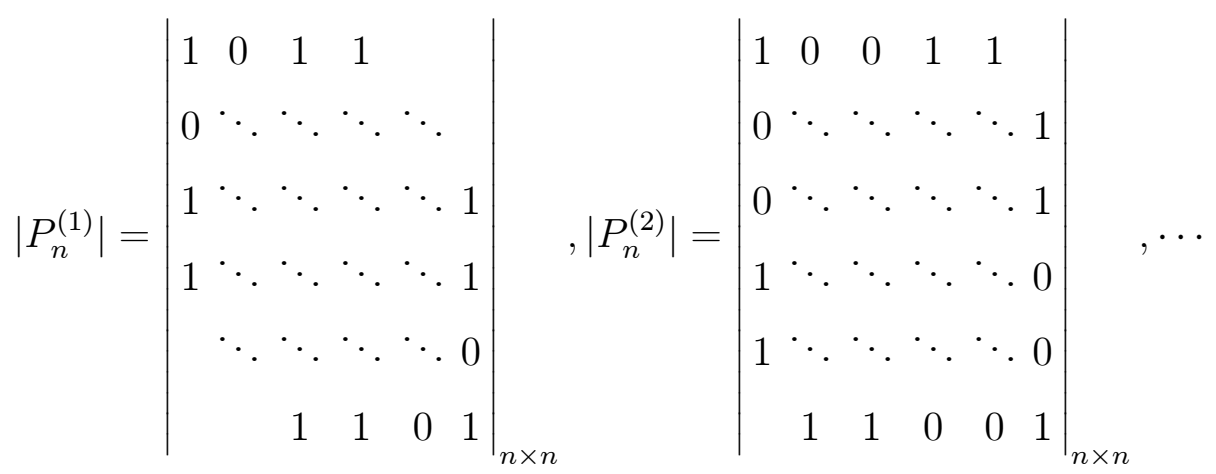

By the software Maple, we obtain their first some terms

\begin{tabular}{|c|c|c|c|c|c|c|c|c|c|c|c|c|c|c|c|c|c|}
\hline$n$ & 5 & 6 & 7 & 8 & 9 & 10 & 11 & 12 & 13 & 14 & 15 & 16 & 17 & 18 & 19 & 20 & 21 \\
\hline$\left|P_{n}\right|$ & 1 & 1 & 0 & 0 & 0 & 1 & 1 & 0 & 0 & 0 & 1 & 1 & 0 & 0 & 0 & 1 & 1 \\
\hline$\left|P_{n}^{(1)}\right|$ & 3 & 7 & 16 & 16 & 0 & 0 & 0 & 81 & 189 & 185 & 176 & -121 & -33 & 775 & 1632 & 3344 & 2048 \\
\hline$\left|P_{n}^{(2)}\right|$ & -1 & 1 & 3 & 5 & 7 & 8 & 4 & 0 & -1 & 1 & 3 & 9 & 15 & 16 & 9 & 5 & 1 \\
\hline$\left|P_{n}^{(3)}\right|$ & 0 & -1 & 1 & 0 & 0 & 0 & 1 & 7 & 24 & 36 & 0 & -9 & -3 & 8 & 35 & 75 & 135 \\
\hline$\left|\widetilde{P}_{n}\right|$ & -2 & 3 & 0 & -3 & 4 & 0 & -4 & 5 & 0 & -5 & 6 & 0 & -6 & 7 & 0 & -7 & 8 \\
\hline$\left|\widetilde{P}_{n}^{(1)}\right|$ & 2 & 0 & 0 & 0 & 2 & 3 & 0 & 0 & 0 & 3 & 4 & 0 & 0 & 0 & 4 & 5 & 0 \\
\hline$\left|\widetilde{P}_{n}^{(2)}\right|$ & 0 & -1 & 2 & 0 & 0 & 0 & 0 & 0 & -2 & 3 & 0 & 0 & 0 & 0 & 0 & -3 & 4 \\
\hline$\left|\widetilde{P}_{n}^{(3)}\right|$ & 0 & 0 & 0 & 1 & 2 & 0 & 0 & 0 & 0 & 0 & 0 & 0 & 2 & 3 & 0 & 0 & 0 \\
\hline
\end{tabular}

where $\left|\widetilde{P}_{n}\right|,\left|\widetilde{P}_{n}^{(1)}\right|,\left|\widetilde{P}_{n}^{(2)}\right|,\left|\widetilde{P}_{n}^{(3)}\right|$ are the corresponding hollow determinants $\left|P_{n}\right|,\left|P_{n}^{(1)}\right|,\left|P_{n}^{(2)}\right|,\left|P_{n}^{(3)}\right|$.

For Heptadiagonal matrix $H D_{n}$, its determinant is equal to

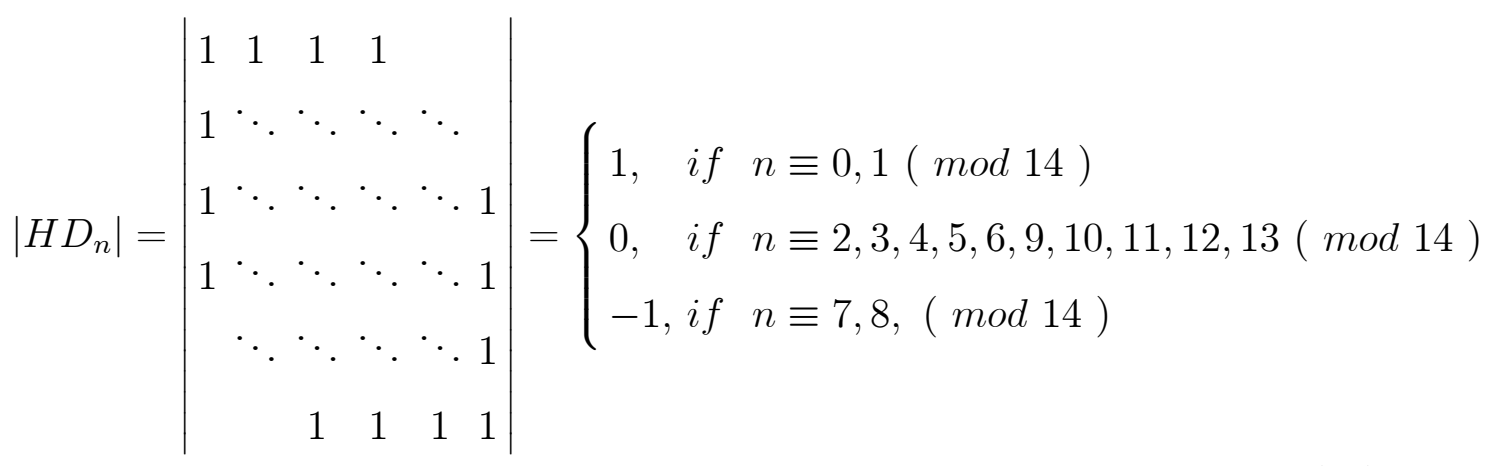




\subsection{The nonsymmetric pentadiagonal Toeplitz matrices}

Theorem 7 For all $n \geq 4$, the determinant of the pentadiagonal Toeplitz matrix containing one element in the subdiagonal above the main diagonal is given by

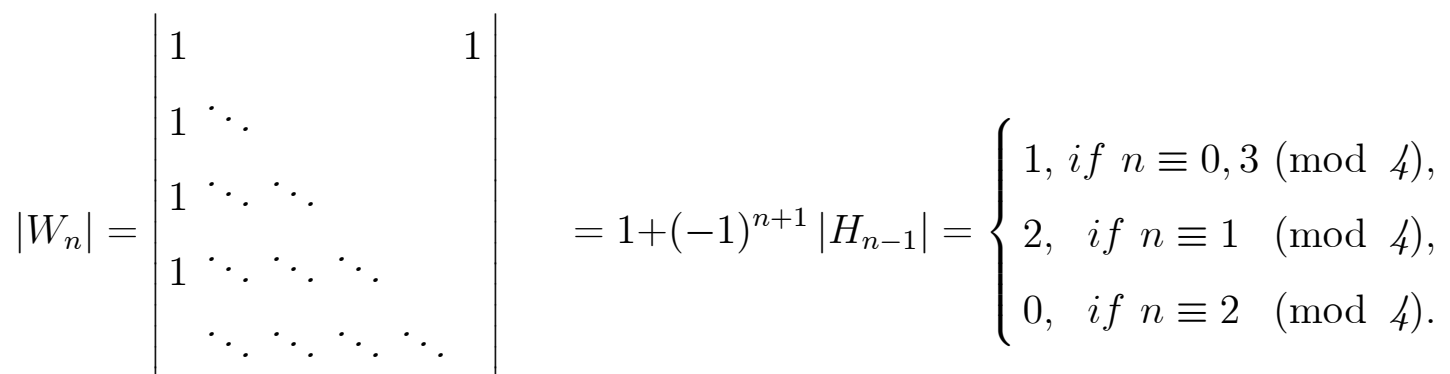

$$
\begin{aligned}
& \left.\begin{array}{llll}
1 & 1 & 1 & 1
\end{array}\right|_{n \times n}
\end{aligned}
$$

PROOF. For all $n \geq 4$, we expand the determinant $\left|W_{n}\right|$ via the Laplace expansion formula with respect to the first row, and obtain

$$
\left|W_{n}\right|=1+(-1)^{n+1}\left|H_{n-1}\right|
$$

Applying the formulas (7), we obtain

$$
\left|W_{n}\right|=1+(-1)^{n+1}\left(\frac{1}{2}+\left(\frac{1}{4}-\frac{i}{4}\right) i^{n-1}+\left(\frac{1}{4}+\frac{i}{4}\right)(-i)^{n-1}\right) .
$$

This finished the proof.

Theorem 8 For all $n \geq 4$, the determinant of the pentadiagonal Toeplitz matrix containing two elements in the subdiagonal above the main diagonal is given by

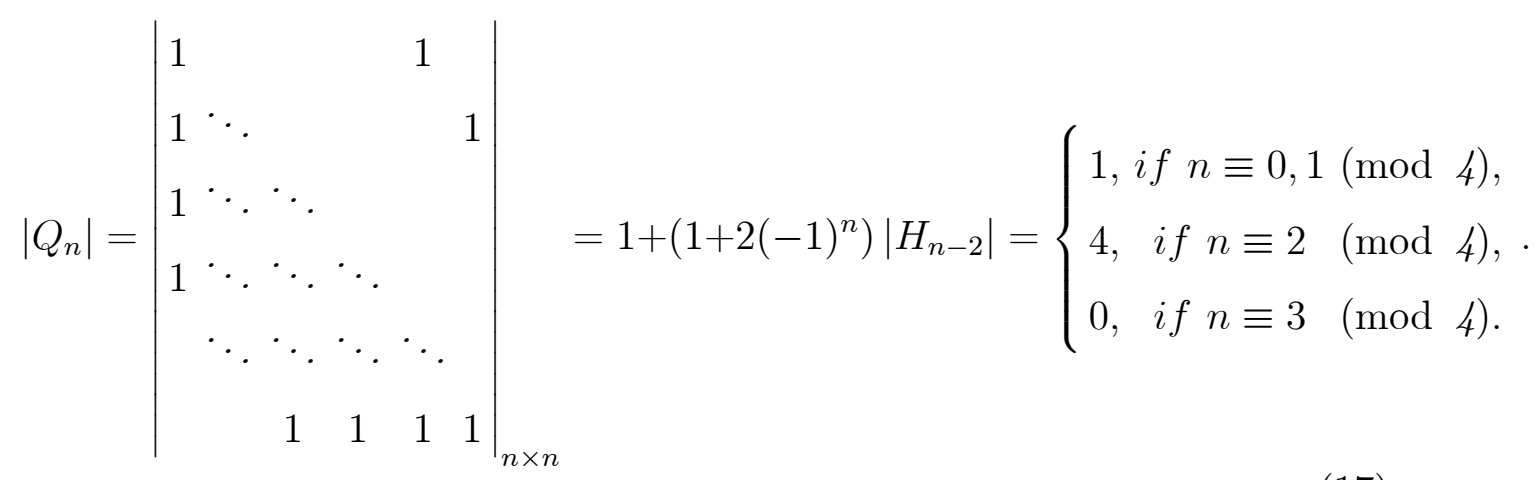


PROOF. For all $n \geq 4$, we expand the determinant $\left|Q_{n}\right|$ in terms of the first row, and obtain

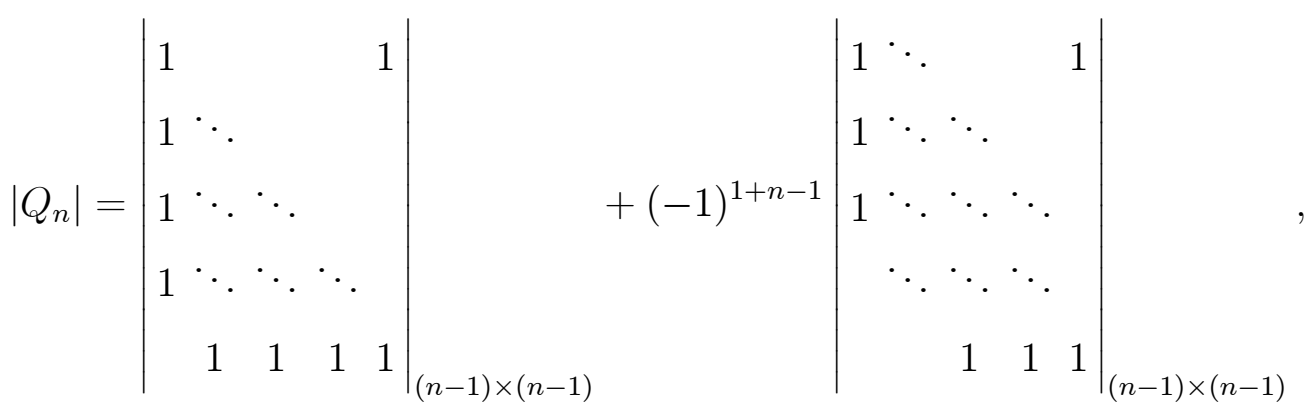

The first determinant is $\left|W_{n-1}\right|$, for the second determinant, we expand it by the last column, and obtain

$$
\left|Q_{n}\right|=\left|W_{n-1}\right|+(-1)^{n}\left(\left|H_{n-2}\right|+(-1)^{1+n-1}\left|H_{n-2}\right|\right)=1+\left(1+2(-1)^{n}\right)\left|H_{n} 2\right|
$$

Using the formulas (6), (7), we obtain the result.

Theorem 9 For all $n \geq 4$, the determinant of the pentadiagonal Toeplitz matrix containing three elements in the subdiagonal above the main diagonal is given by

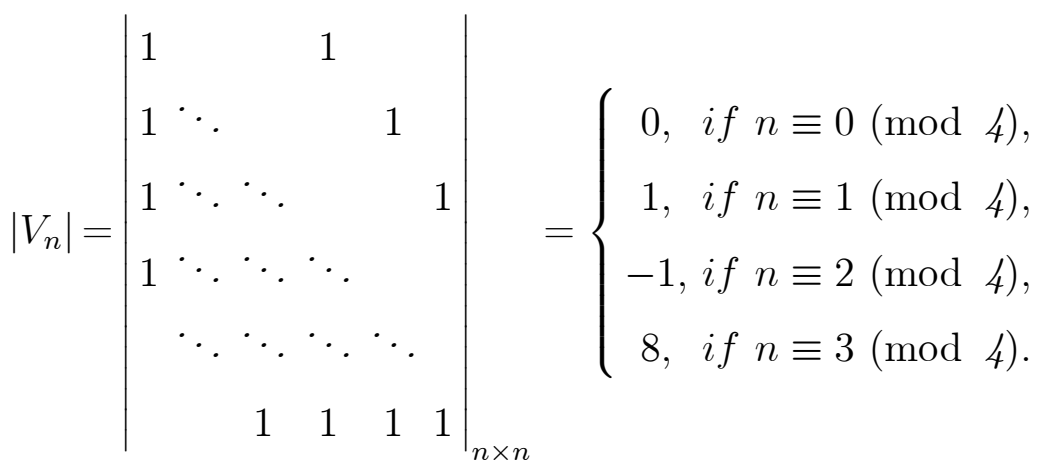

$$
\begin{aligned}
& =1+(-1)^{n-1}+3\left(1+(-1)^{n-1}\right)\left|H_{n-3}\right|-\left|H_{n-5}\right| \text {. }
\end{aligned}
$$

PROOF. For all $n \geq 4$, we expand the determinant $\left|V_{n}\right|$ in terms of the first row, and obtain that the first determinant is $\left|Q_{n}{ }_{1}\right|$. For the second determinant, we expand it by the last column, and obtain 


$$
\left|V_{n}\right|=\left|Q_{n-1}\right|+(-1)^{n-1}\left|H_{n-3}\right|+\left|H_{n-3}\right|+\left|\begin{array}{rrrrr}
1 & 1 & & & \\
1 & \ddots & \ddots & \ddots & \\
\ddots & \ddots & \ddots & \ddots & \\
& \ddots & \ddots & \ddots & \\
& \ddots & \ddots & 1 \\
& & 1 & 1
\end{array}\right|_{(n-2) \times(n-2)}
$$

Thus, using aforementioned notations and the formula (6) and (7), we obtain

$$
\left|V_{n}\right|=1+(-1)^{n-1}+3\left(1+(-1)^{n-1}\right)\left|H_{n-3}\right|-\left|H_{n-5}\right| .
$$

This finishes the proof.

\subsection{The nonsymmetric generalized Toeplitz matrices}

By similar arguments, we obtain two results from nonsymmetric structure six-diagonal Toeplitz determinants, whose two subdiagonals above the main diagonal containing three and five elements, respectively. The proofs have been omitted.

Theorem 10 For all $n \geq 4$, the determinant of the six-diagonal Toeplitz matrix containing three elements in the two consecutive subdiagonal above the main diagonal is given by

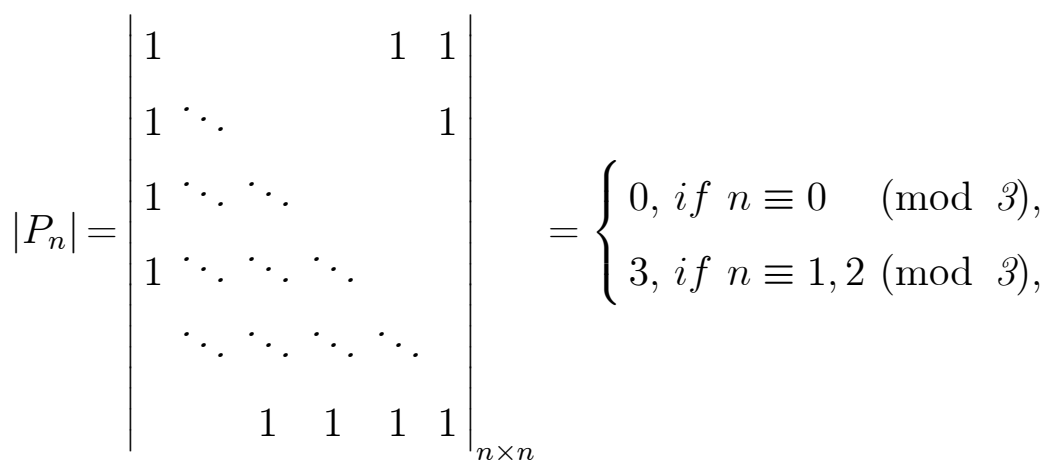

$$
\begin{aligned}
& =2+2(-1)^{n}\left|T_{n-2}^{(1)}\right|+(-1)^{n+1}\left|T_{n-1}^{(1)}\right| \text {. }
\end{aligned}
$$

Theorem 11 For all $n \geq 4$, the determinant of the six-diagonal Toeplitz matrix containing five elements in the two consecutive subdiagonal above the main diagonal is given by 


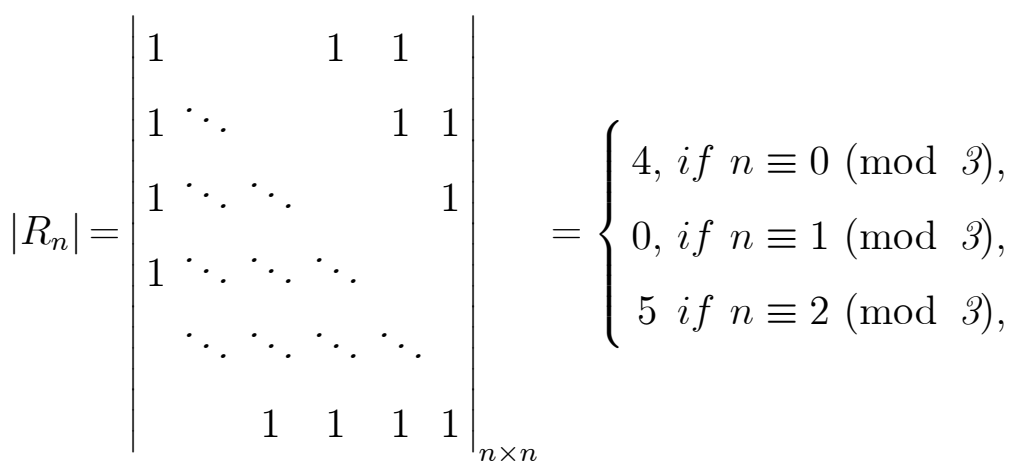

$$
\begin{aligned}
& =3+2(-1)^{n}\left|T_{n-1}^{(1)}\right|+(-1)^{n-1}\left|T_{n-3}^{(1)}\right| \text {. }
\end{aligned}
$$

The relations (16)-(20), (21)-(22) show that expansions (2) can be derived from the Laplace expansion, the combinatorial coefficients may various integers, not only $-1,0,1$.

\section{Some studies on the results [9]}

In [5], the author defined an $n \times n$ lower Hessenberg Toeplitz matrix $F_{n}$ by

$$
f_{i, i-k}=\left\{\begin{array}{l}
1, k \in\{-1,0,2,4,6, \cdots \mid i-k>0\} \\
0, \quad \text { otherwise. }
\end{array}\right.
$$

and proved that it was the maximum absolute determinants of upper Hessenberg Bohemian whose elements from $\{0,1\}$.

We define an $n \times n$ lower Hessenberg Toeplitz matrix $D_{n}$ by

$$
d_{i, i-k}=\left\{\begin{array}{cc}
1, & k \in\{-1,0,2,4,6, \cdots \mid i-k>0\} \\
-1, & \text { otherwise }
\end{array}\right.
$$

Applying Lemma 1, with the initial values $\left|D_{0}\right|=\left|D_{1}\right|=1$, we obtain a recurrence relation

$$
\left|D_{n}\right|=\left|D_{n-1}\right|+\left|D_{n-2}\right|+\cdots+\left|D_{1}\right|+\left|D_{0}\right|, \text { for } n>1
$$

and an explicit formula of the determinant,

$$
\left|D_{n}\right|=2^{n-1}, \text { for } n \geq 1
$$


Define an $n \times n$ lower Hessenberg Toeplitz matrix $\widetilde{D}_{n}([9]$, let $d=1)$ by

$$
\begin{gathered}
\tilde{d}_{i, j}=\left\{\begin{array}{cc}
0, & i>j+1, \\
1, & i=j+1, \\
-1, & i \leq j
\end{array}\right. \\
\left|\widetilde{D}_{n}\right|=(-1)^{n} 2^{n-1} .
\end{gathered}
$$

We define an $n \times n$ lower Hessenberg Toeplitz matrix $N_{n}$ by

$$
n_{i, i-k}=\left\{\begin{array}{cc}
1, \quad k \in\{-1,0,1,3,5, \cdots \mid i-k>0\} \\
-1, \quad \text { otherwise }
\end{array}\right.
$$

For example, the determinant of $5 \times 5$ lower Hessenberg Toeplitz matrix with elements from the set $\{-1,1\}$ and subdiagonal elements fixed at 1 ,

$$
\left|N_{5}\right|=\left|\begin{array}{ccccc}
1 & 1 & & & \\
1 & 1 & 1 & & \\
-1 & 1 & 1 & 1 \\
1 & -1 & 1 & 1 & 1 \\
-1 & 1 & -1 & 1 & 1
\end{array}\right| .
$$

We obtain that, for $n \geq 2$,

$$
\left|N_{n}\right|=\left\{\begin{array}{ccc}
0, & \text { if } n \equiv 2 & (\bmod 4) \\
(-1)^{\left\lfloor\frac{n-2}{4}\right\rfloor+1} 2^{\left\lfloor\frac{n}{2}\right\rfloor}, & \text { if } n \equiv 3 & (\bmod 4) \\
(-1)^{\left\lfloor\frac{n-2}{4}\right\rfloor+1} 2^{\left\lfloor\frac{n}{2}\right\rfloor}, & \text { if } n \equiv 0 & (\bmod 4) \\
(-1)^{\left\lfloor\frac{n-2}{4}\right\rfloor+1} 2^{\left\lfloor\frac{n}{2}\right\rfloor}, & \text { if } n \equiv 1 & (\bmod 4)
\end{array}\right.
$$

\begin{tabular}{|c|c|c|c|c|c|c|c|c|c|c|c|c|c|}
\hline $\mathrm{n}$ & 5 & 6 & 7 & 8 & 9 & 10 & 11 & 12 & 13 & 14 & 15 & 16 & 17 \\
\hline$\left|F_{n}\right|$ & 5 & 8 & 13 & 21 & 34 & 55 & 89 & 144 & 233 & 377 & 610 & 987 & 1597 \\
\hline$\left|D_{n}\right|$ & $2^{4}$ & $2^{5}$ & $2^{6}$ & $2^{7}$ & $2^{8}$ & $2^{9}$ & $2^{10}$ & $2^{11}$ & $2^{12}$ & $2^{13}$ & $2^{14}$ & $2^{15}$ & $2^{16}$ \\
\hline$\left|\widetilde{D}_{n}\right|$ & $-2^{4}$ & $2^{5}$ & $-2^{6}$ & $2^{7}$ & $-2^{8}$ & $2^{9}$ & $-2^{10}$ & $2^{11}$ & $-2^{12}$ & $2^{13}$ & $-2^{14}$ & $2^{15}$ & $-2^{16}$ \\
\hline$\left|N_{n}\right|$ & -4 & 0 & 8 & 16 & 16 & 0 & -32 & -64 & -64 & 0 & 128 & 256 & 256 \\
\hline
\end{tabular}

By this results, we have three remarks: 
Remark 12 For $n \geq 11$, Proposition 3.6 in [9] is not true.

As showing in the above table, $\left|N_{11}\right|=-32<-20,\left|N_{12}\right|=-64<-22$, $\left|N_{13}\right|=-62<-24 .\left|N_{15}\right|=128>28,\left|N_{16}\right|=256>30,\left|N_{17}\right|=256>32$. They do not satisfy Proposition 3.6.

Although one has found the maximum abstract values of the determinant of Bohemian, the values of the determinants of Bohemian are not continued, for instance, formulae (16) - (22) and the determinants $\left|F_{n}\right|,\left|N_{n}\right|,\left|D_{n}\right|,\left|\widetilde{D}_{n}\right|$. Thus, we have

Remark 13 The propositions 3.7 and 3.8 in [9] are valueless of the enumerations of distinct determinants of Bohemians.

Remark 14 Conjecture 18, 19, and 20 [25] can not be proved by Proposition 3.6, 3.7 and $3.8[9]$.

\section{References}

[1] G.E. Bergun, V.E. Hoggatt Jr., A family of tridiagonal matrices, Fibonacci Quart. 16 (1978)285-288.

[2] N. D. Cahill, J. R. D'Errico, D. A. Narayan, J. Y. Narayan, Fibonacci determinants, The college mathematics Journal, 33(2002)221-225.

[3] E.Y.S. Chan, R. M. Corless, Laureano G.-V., J. R. Sendra, J. Sendra, E. S. Thornton. Upper Hessenberg and Toeplitz Bohemians. Linear Algebra Appl., 601(2020)72-100.

[4] E.Y.S. Chan, R. M. Corless, Laureano G.-V., J. R. Sendra, J. Sendra, S. E. Thornton. Bohemian upper Hessenberg. CoRR, abs,(2018)/1809.10653.

[5] L. Ching. The maximum determinant of an $n \times n$ lower Hessenberg $(0,1)$ matrix. Linear Algebra Appl., 183(1993)147-153.

[6] Z. Cinkir. An elementary algorithm for computing the determinant of pentadiagonal Toeplitz matrices. J. Comput. Appl Math. 236(2012)2298-2305.

[7] L. Du, T. Sogabe, S.-L. Zhang. An algorithm for solving nonsymmetric pentadiagonal Toeplitz linear systems. Appl. Math.Comput. 244(2014)10-15.

[8] G. Everest, A. van der Poorten, I. Shparlinski, and T. Ward. Recurrence Sequences. American Mathematical Society, 2003.

[9] M. Fasi, G. M. N. Porzio, Determinants of normalized bohemian upper Hessenberg matrices. Elec. J. Linear Alg. 36(2020)352-366.

[10] J. Feng, Hessenberg Matrices On Fibonacci And Tribonacci Numbers. Ars Combinatoria, 127(2016)117-124. 
[11] J. Feng, Hongtao Fan. Results on determinants of specific pentadiagonal Toeplitz and Hessenberg matrices. Appl. Math.Comput. (under review).

[12] C.M. Fonseca, F. Yllmaz. Some comments on k-tridiagonal matrices: determinant, spectra, and inversion. Appl Math Comput. 270(2015)644-647.

[13] G.H. Golub, C.F. Van Loan, Matrix Computations, Fourth ed.,Johns Hopkins University Press, Baltimore, MD, 2013.

[14] T. Goy, M. Shattuck. Determinant identities for Toeplitz-Hessenberg matrices with tribonacc number entries. arXiv: 2003.10660.

[15] ADA Hadj, M. Elouafi. On the characteristic polynomial, eigenvectors and determinant of a pentadiagonal matrix. Appl Math Comput. 198(2008)634-642.

[16] M. M .Jeffrey. A fast algorithm for solving diagonally dominant symmetric pentadiagonal Toeplitz systems. J. Comput. Appl. Math. 234(2010)995-1005.

[17] J. Jia, B. Yang, S. Li. On a homogeneous recurrence relation for the determinants of general pentadiagonal Toeplitz matrices. Comput Math Appl. 71(2016)1036-1044.

[18] J. Jia, Y. Jiang. A structure preserving matrix factorization for solving general periodic pentadiagonal Toeplitz linear systems, Comput Math Appl. 66(2013)965-974.

[19] E. Kilic, M. El-Mikkawy. A computational algorithm for special nth-order pentadiagonal Toeplitz determinants, Appl. Math.Comput. 199(2008)820-822.

[20] X. G. Lv, TZ Huang, J. Le. A note on computing the inverse and the determinant of a pentadiagonal matrix. Appl Math Comput. 206(2008)327-331.

[21] R. B. Marr, G. H. Vineyard. Five-diagonal Toeplitz determinants and their relation to Chebyshev polynomials. SIAM J Matrix Anal Appl. 9(1988) 579586 .

[22] A. Milica, C. M. da Fonseca, Some determinantal considerations for pentadiagonal matrices, Linear and Multilinear Algebra, (2020) https://doi.org/10.1080/03081087.2019.1708845.

[23] S. S. Nemani. A fast algorithm for solving Toeplitz penta-diagonal systems, Appl. Math.Comput. 215(2010)3830-3838.

[24] R. A. Sweet. A recursive relation for the determinant of a pentadiagonal matrix. Comm ACM. 12(1969)330-332.

[25] S. E. Thornton, The Characteristic Polynomial Database, published electronically at http://bohemianmatrices.com/cpdb, 06.12.2020.

[26] S. E. Thornton, Algorithms for Bohemian Matrices (2019). Electronic Thesis and Dissertation Repository. https://ir.lib.uwo.ca/etd/6069. 